\title{
Kerala Mural Paintings: Diversification and Application in Home, Apparel and Accessories
}

\author{
Akshaya A $S^{1}$, Dhanashree $S^{2}$, Dharsana $S^{3}$, Dhivya $R^{4}$, Hema Sri $R^{5}$ and Dr. \\ Mariyam Adnan ${ }^{6}$ \\ 1,2,3,4,5Students, M.Sc. Fashion Design \& Merchandising, Department of Apparel \& Fashion \\ Design, PSG College of Technology, Coimbatore, India. \\ ${ }^{6}$ Assistant Professor (Selection Grade), Department of Apparel E Fashion Design, PSG \\ College of Technology, Coimbatore, India. ORCID: oooo-0oo2-8327-2751. \\ Email:mag.afd@psgtech.ac.in
}

\begin{abstract}
A study on diversification of Kerala mural paintings was conducted by making different variety of products with different styles of design. Rather than confining Kerala murals on wall, nowadays several mediums are used to increase its aesthetic value and attract the potential customers. In this paper, the development of traditional and contemporary mural products is discussed. A survey was conducted first to know about the preferences of the potential customers on Kerala Mural products and products to be produced were selected according to this survey. Later, another survey was conducted to test the visual appearance and price range of the developed mural products. The study concludes by learning that people are showing interest in technological advancements in mural painting and its diversification for special purposes like social issues.
\end{abstract}

Keywords: Kerala Mural Painting, Wall hangings, Laptop pouches, Shawl, Diversification

\section{Introduction:}

Mural painting refers to those paintings that are done on wall (Mini, P.V, 2010). These mural paintings contain priceless information about our past and cultural heritage. Since they always depict sceneries of life and religious traditions, they hold a great deal of significance to mankind (Nimisha, P, 2017). The origin of mural paintings dates back to $2^{\text {nd }}$ century BC in the caves Ajanta, Bagh and Sithanavasal. At first the mural paintings were in the form of rock engravings which can be seen even today in the caves of Edkkal, Wayand. Later it got transformed into rock paintings that can be seen at Anjanad valley, Idukki district (Mini, P.V, 2010). Thus the tradition of wall painting got introduced. The evolutions of these mural paintings are now seen as the different forms of mural painting all over India. These evolved mural paintings include Pithora and Kamangari wall paintings of Gujarat; Thangka painting of Himachal Pradesh; Madhubani painting of Bihar; Aipan painting of Uttarkhand; Saora painting of Orissa; Badami painting of Karnataka; Mandana, Sanjhi, Thapa, Bhil, Rajput and Fresco of Rajasthan; Tanjore and Sithanavasal of Tamil Nadu; Sohrai and Khovar of Jharkhand; Warli art of Maharastra; Manuscript art of BhagavataPurana of Assam and Kerala mural.

(c) AesthetixMS 2020. This Open Access article is published under a Creative Commons Attribution Non-Commercial 4.0 International License (http://creativecommons.org/licenses/by-nc/4.0/), which permits non-commercial re-use, distribution, and reproduction in any medium, provided the original work is properly cited. For citation use the DOI. For commercial re-use, please contact editor@chitrolekha.com 


\section{Kerala mural painting}

Kerala mural painting was first discovered in a shrine at Thirunandikkara in the $8^{\text {th }}$ century, which is now situated in KanyaKumari district. The unique feature that distinguishes Kerala mural painting from the other forms is its vibrancy and elegance. The history of Kerala Mural painting started from the discovery of rock engravings in the caves of Edkkal in Wayanad district and Perimkadavila in Thiruvananthapuram district of Kerala followed by pre-historic rock paintings found in the Anjanad valley of Idukki district.

\section{Motifs of Kerala mural paintings}

Usually Kerala mural paintings depict Hindu mythology and there are certain motifs that can be seen on all these paintings. These motifs add a traditional touch to these mural paintings. Some of the prominent motifs of Kerala mural paintings are Mazhipookal, Nazhikamali and Veeralipattu as shown in Fig. 2.

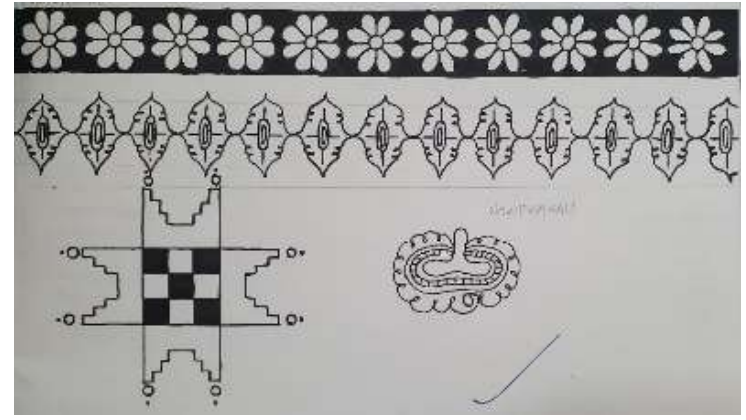

Fig. 2: Motifs of Kerala mural painting

\section{Techniques of Kerala mural paintings}

The technique of Kerala mural painting is quite simple but at the same time, is a time consuming process. There are six stages in Kerala mural painting. First stage is the sketching a design on a white fabric. The second stage is enhancing the outline of the sketch. Third stage is colouring the painting. The only step that needs to be followed while painting a Kerala mural is the order of applying the Panchavarna colours (yellow, red, green, blue). Fourth stage is applying gradation. Fifth stage is the application of black outline. Sixth stage is the final and finishing touches are done to the painting (Sandhya Ravi, 2015).

\section{Materials and Methods:}

\section{Materials}

The materials used are Acrylic paints, Medium 1 (prevents spreading when mixed with paints), Fabric, Fasteners - Velcro, zipper, magnetic buttons, plastic buttons, Paint brush, Stationery items such as pencil, CD marker, eraser, sharpener and A3 paper.

\section{Methods}

Research methodology consists of the number of procedures or techniques used to identify, select, process, and analyse information about the craft. The flowchart in Fig.1 shows the methodology adopted for Kerala mural painting. 


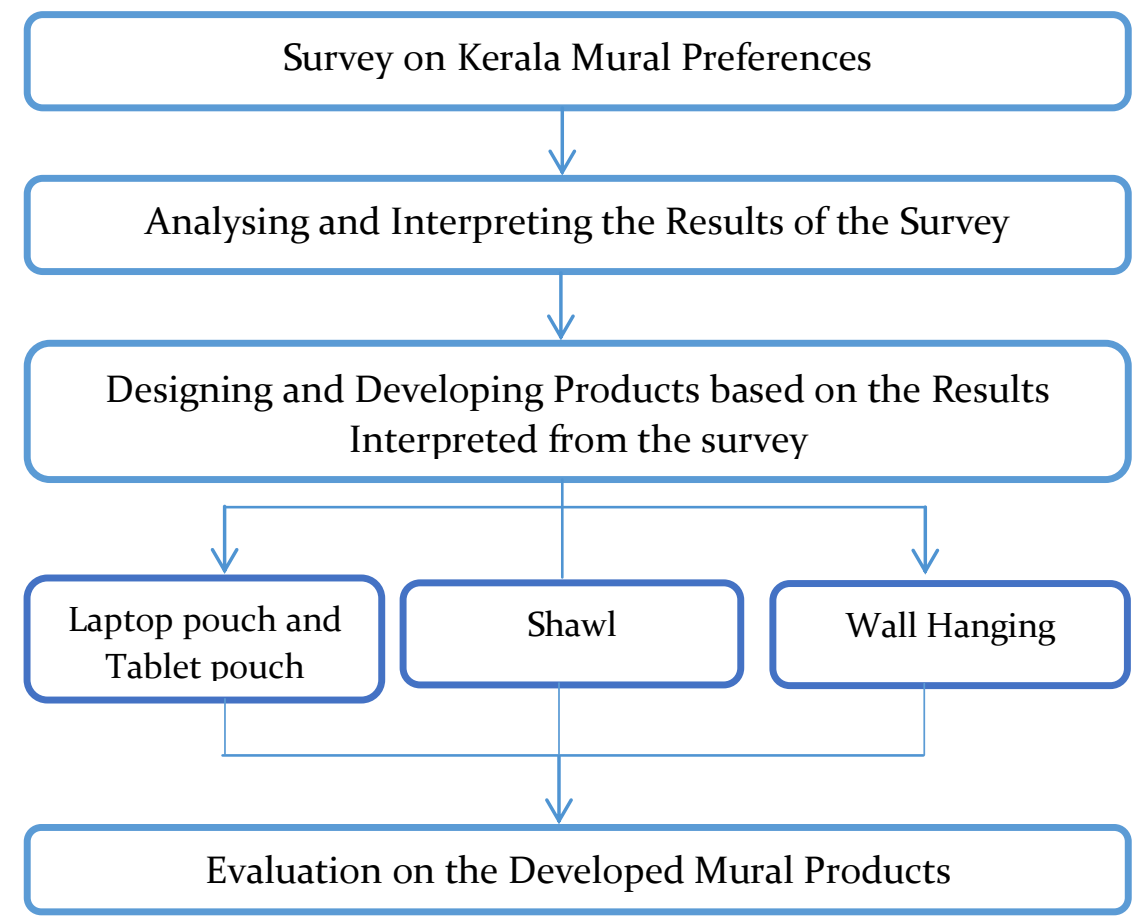

Fig. 1: Methodology of products developed by Kerala Mural painting

\section{Survey on Kerala mural preferences}

A survey was conducted to learn about the preferences of the potential customers and to develop products based on the results of the survey. 68 respondents within the age group of 20 and above were surveyed.

\section{Sample Questionnaire}

We are conducting this survey to know about your preferences for Kerala mural products. We would like you to share your opinions about the following questions related to Kerala Mural paintings.

Table 1: Sample questionnaire on preferences of Kerala murals

1) Are you aware of Kerala Mural paintings?
a) Yes
b) No

2) Are you aware that the mural painting is different in different states in India?

\begin{tabular}{|l|l}
\hline a) Yes & b) No \\
\hline
\end{tabular}

3) The type of design you want in mural painting?
a) Contemporary
b) Traditional 
c) Fusion

4) Which mural product would you prefer to buy?

\begin{tabular}{|l|l|}
\hline a) Wall hanging & b) Garment \\
\hline c) Laptop pouch & d) Jewellery
\end{tabular}

5) Are you interested in the diversification of Kerala Mural paintings for special purpose?
a) Yes
b) No

The results of the survey were analysed thoroughly and interpreted into useful data that help in the development of innovative mural products.

\section{Evaluating the Developed Mural Products through questionnaire}

After developing the mural products, a survey was conducted on the products to evaluate the visual appearance and price range of the products developed.

\section{Sample Questionnaire}

We have designed and developed mural products based on the previous survey conducted to know the preference of the potential customers.

Table 2: Sample questionnaire for developed products

1) Rate the following in terms of visual appearance.

Laptop pouch/ Tablet pouch

$\begin{array}{llll}\text { Poor Fair Good } \quad \text { Very good } & \text { Excellent }\end{array}$

Shawl

Poor Fair Good Very good Excellent

Wall hanging

$\begin{array}{lllll}\text { Poor } & \text { Fair } & \text { Good } & \text { Very good }\end{array}$

2) Did you like the concept of fusion of Warli art and Kerala art paintings in laptop pouch?
a) Yes
b) No

3) Where do you prefer to buy mural products from?
a) Retail store
b) Online website
c) Artist

4) How much will you be willing to pay for the following products? 
5 | Kerala Mural Paintings: Diversification and Application in Home, Apparel and Accessories

\begin{tabular}{|c|l|l|}
\hline \multicolumn{2}{|l|}{ Laptop pouch/ Tablet pouch } & b) 1000-1500 \\
\hline a) $700-1000$ & b) $700-1000$ & c) $1000-1200$ \\
\hline Shawl & \multicolumn{2}{|l|}{} \\
\hline a) $500-700$ & b) $700-1000$ & c) $1000-1500$ \\
\hline Wall hanging & & \\
\hline a) $500-700$ &
\end{tabular}

\section{Results and Discussion}

\section{Analysing and interpreting the results of the survey}

The results of the survey on Kerala mural preferences are as follows:

According to the survey, about $72.1 \%$ of people were already aware of the existence of different mural paintings across India (Fig. 3).

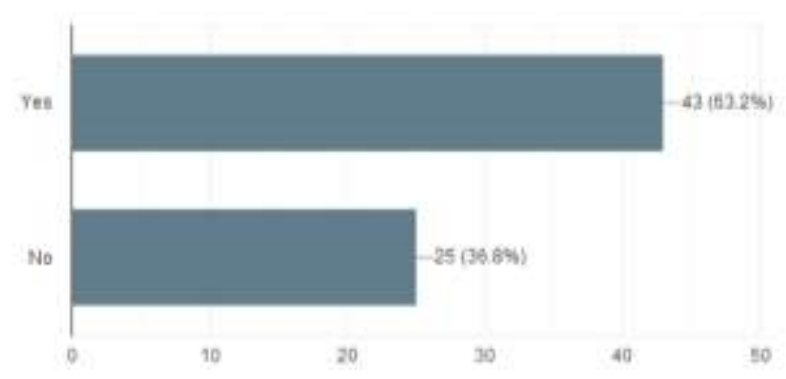

Fig. 3: Awareness of Kerala Mural painting

Only $63.2 \%$ of people were aware of Kerala mural painitngs (Fig. 4).

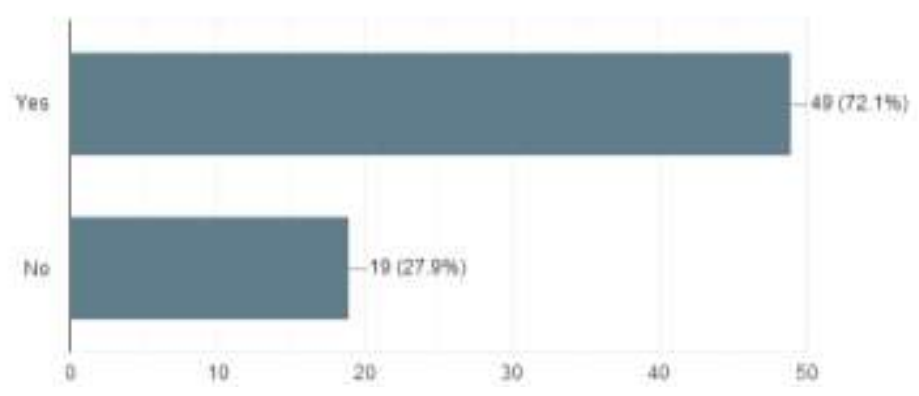

Fig. 4: Awareness of different Mural paintings across India 
Based on the survey, $51.5 \%$ of people prefered traditional mural paintings, $44.1 \%$ of people prefered contemporary style of mural paintings, while $42.6 \%$ of people prefered fusion of different types of mural paintings as shown in Fig. 5.

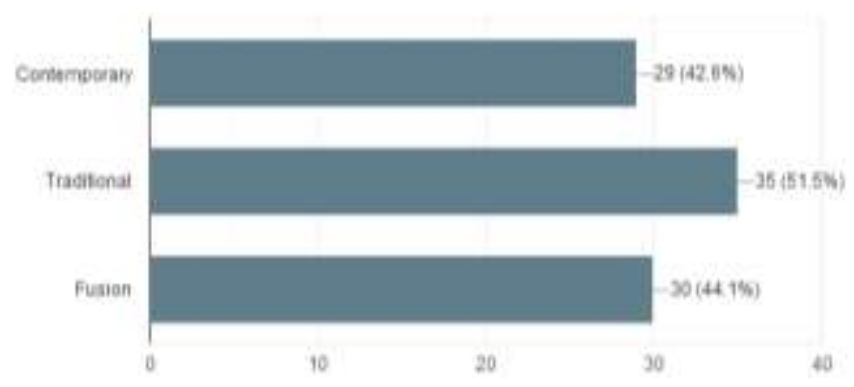

Fig. 5: Selection of design

Based on the survey, about $77.9 \%$ of people prefered to buy mural paintings done on wall hanging followed by garments (16.2\%), jewellery (14.7\%) and laptop pouch $(13.2 \%)$ as shown in Fig. 6.

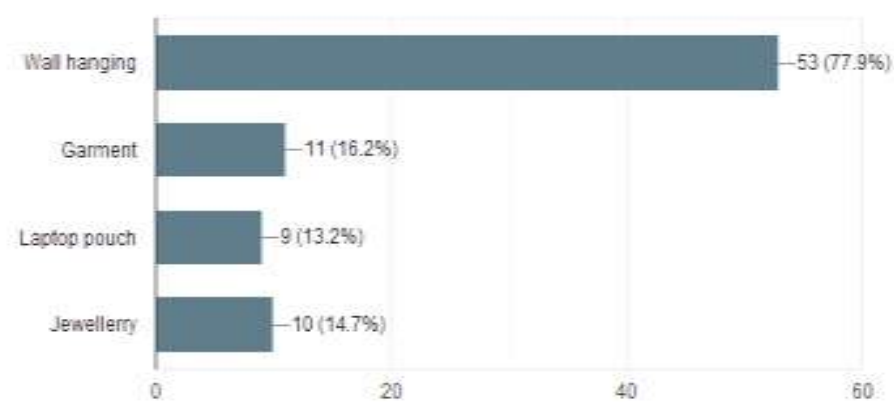

Fig. 6: Preference of mural product

The result also showed that about $85.3 \%$ of people were interested in the diversification of Kerala Mural painting for special purposes as shown in Fig. 7 .

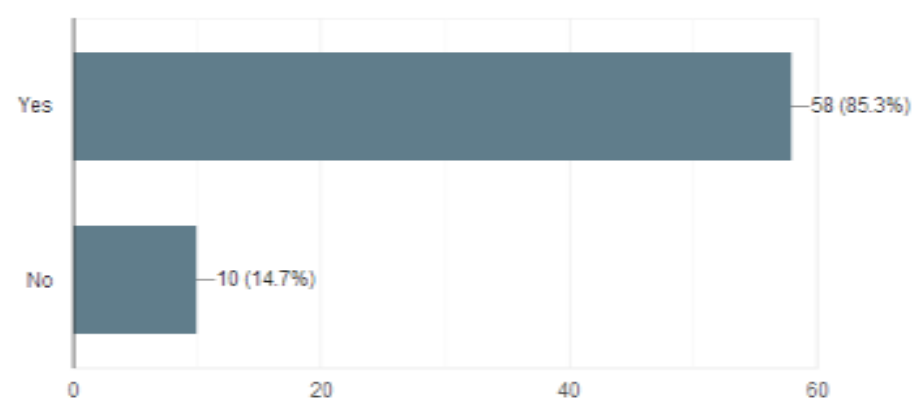

Fig. 7: Diversification of Kerala mural painting 


\section{Innovative product development using Kerala mural painting}

Based on the results of the survey on preferences of Kerala mural paintings, three categories of products with 5 different design ranges were developed.

The product to be developed were Laptop pouch and Tablet pouch, Shawl and Wall hanging.

\section{Process Sequence for developing Laptop pouch}

Fabrics were cut according to the dimensions of the laptop. Design was traced on the fabric by drawing with a pencil as shown in Fig 8.

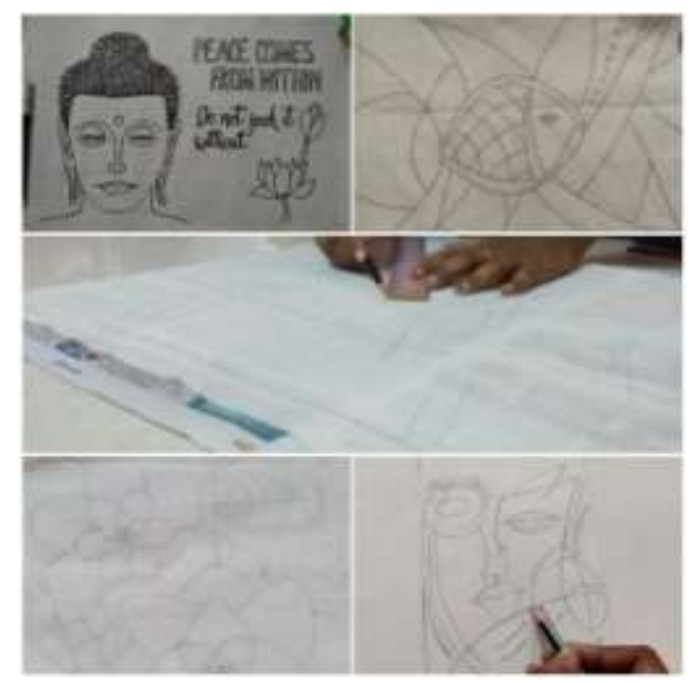

Fig. 8: Design template

Yellow colour was first applied among the other colours following the order of Panchavarna from the traditional Kerala mural technique. Second coating of yellow colour was given as shown in Fig.9.

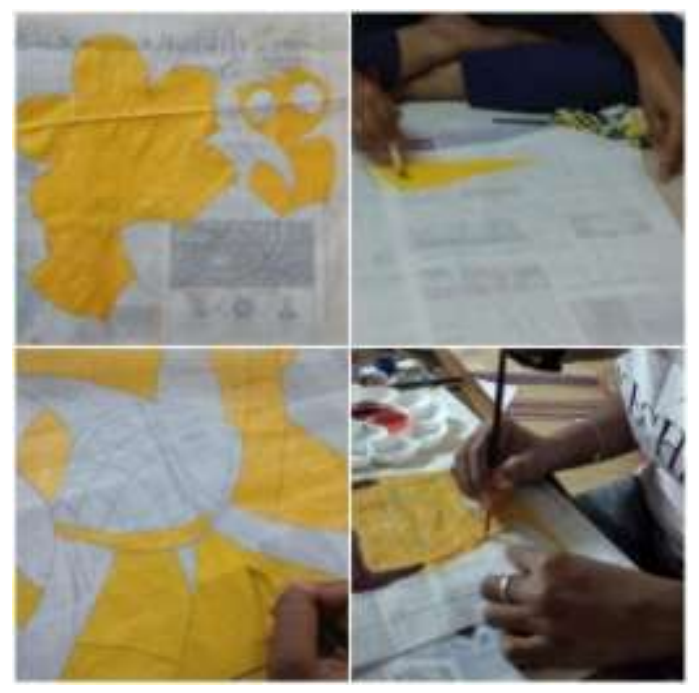

Fig. 9: Yellow coating of the design

Red colour was applied next followed by blue and green. Other shades and tints of Panchavarna were also added depending on the design variations as shown in Fig. 10. 


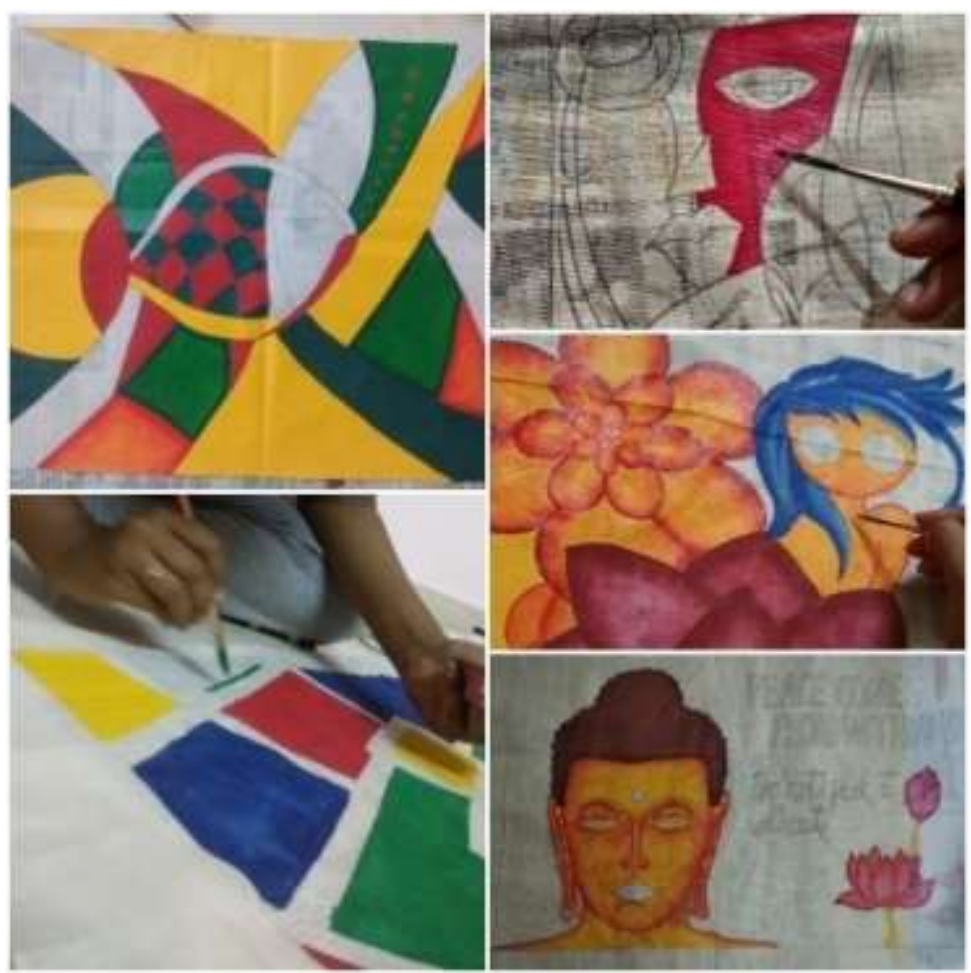

Fig. 10: Applying colours in the order of Panchavarna

Gradation was done depending on the design requirement as shown in Fig. 11. Final corrections were done.
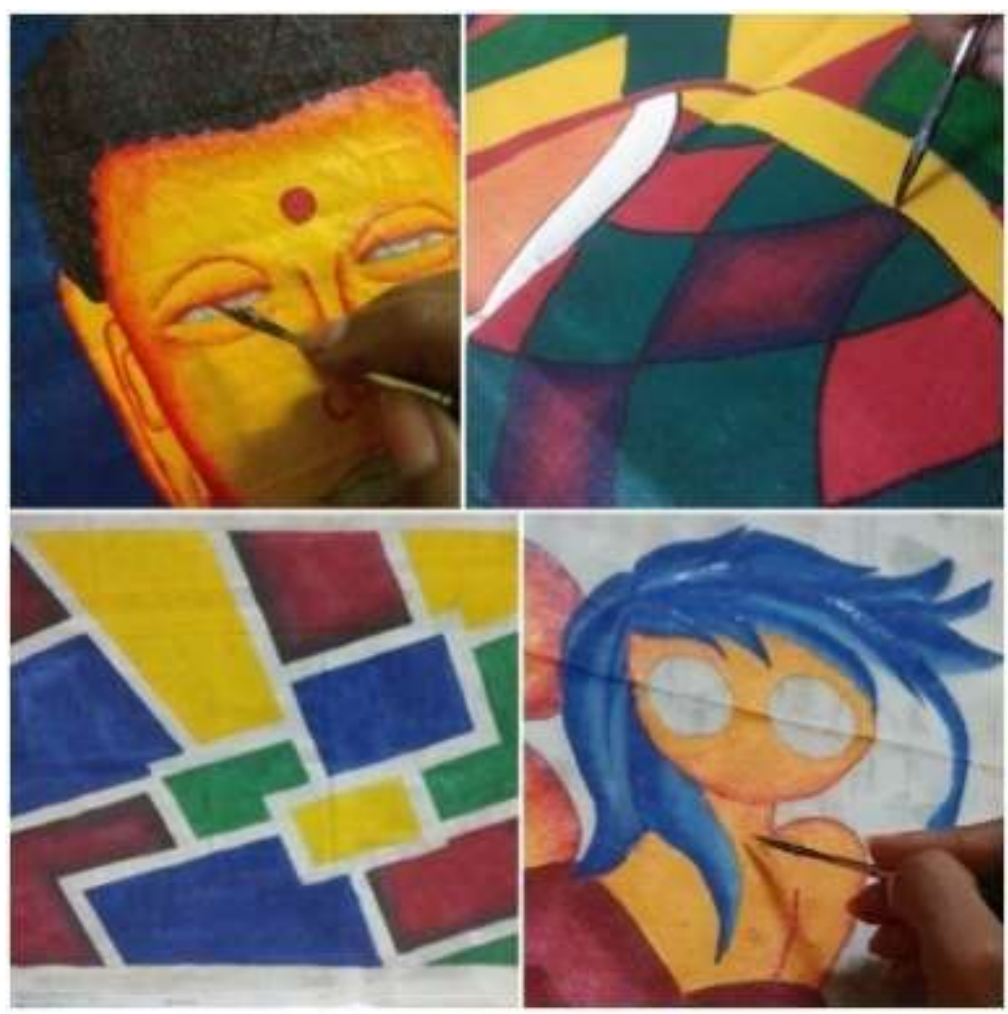

Fig. 11: Gradation done in the required places of the design 
9 | Kerala Mural Paintings: Diversification and Application in Home, Apparel and Accessories

Last step was drawing the outline of the design using black colour as shown in Fig. 12.

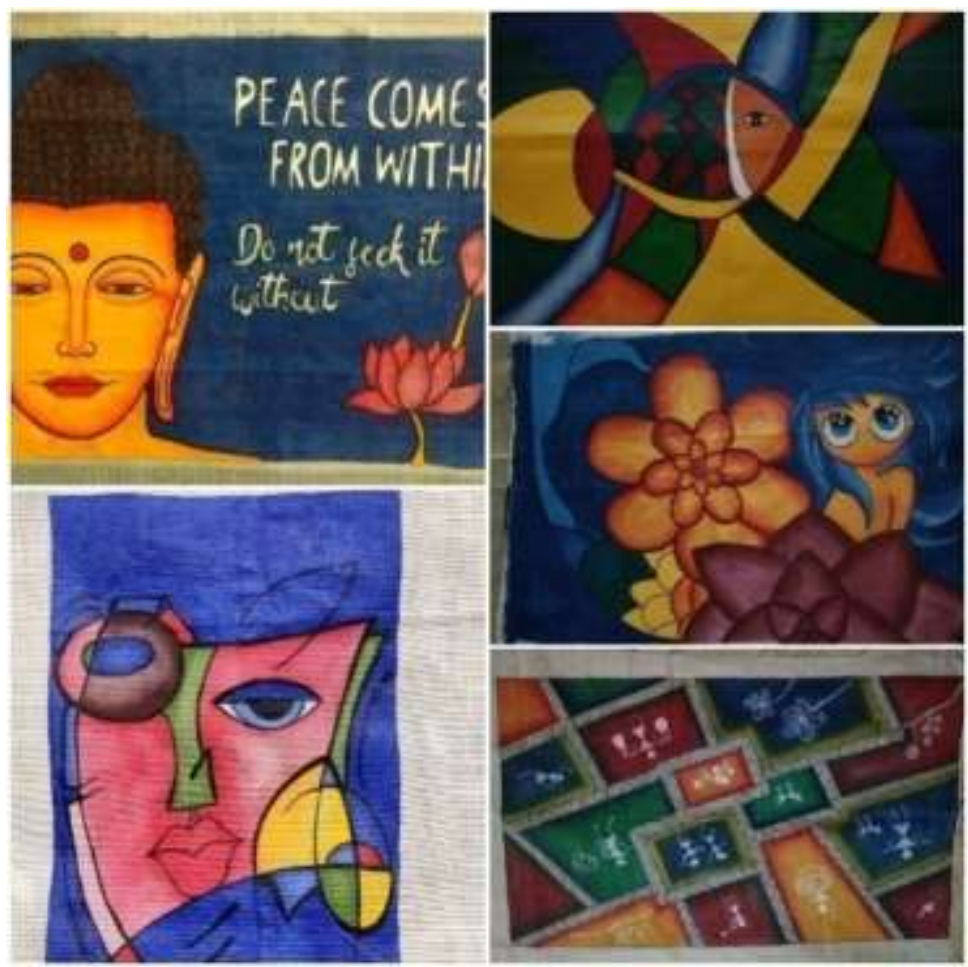

Fig. 12: Final painting before stitching

The painted fabrics were then ironed and converted into laptop pouches and tab pouch.

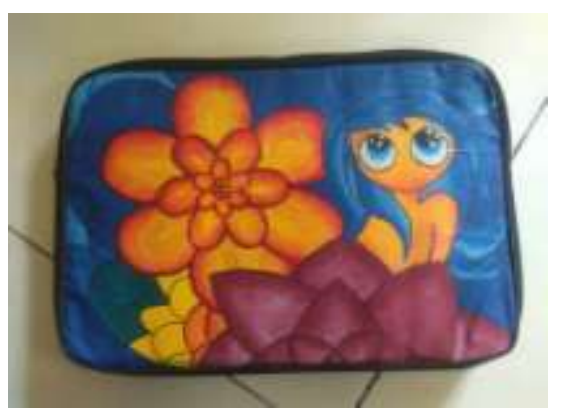

A

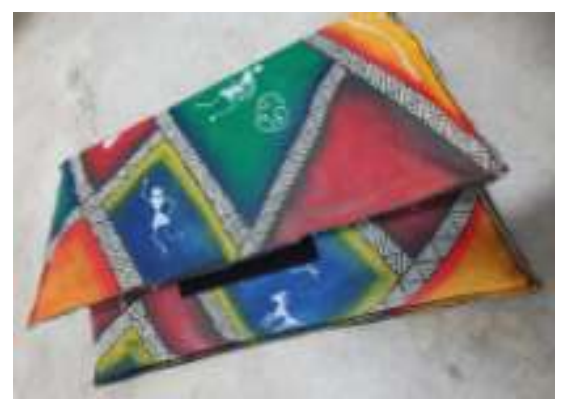

C

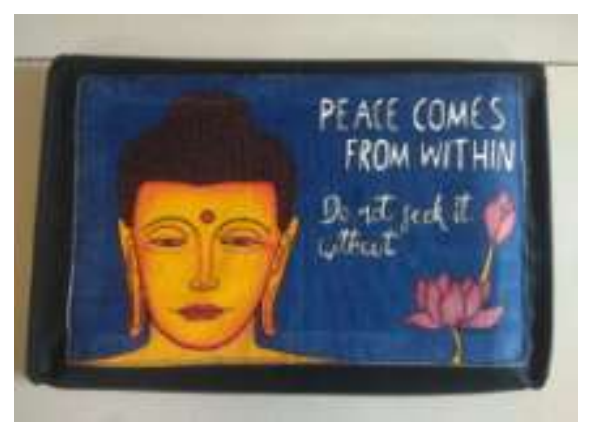

B

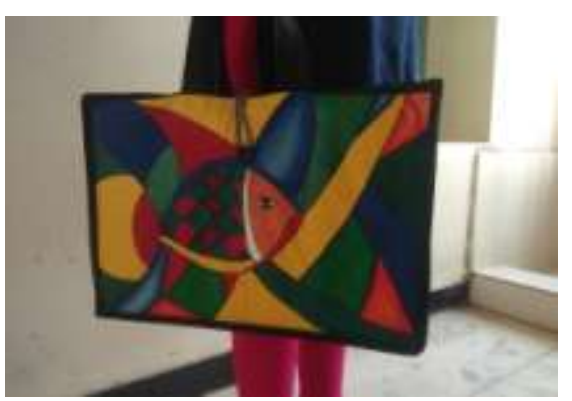

D 


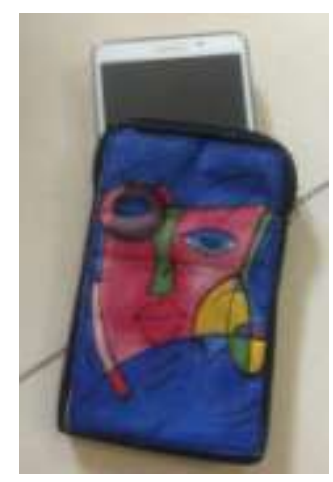

$\mathbf{E}$

Fig. 13: Developed laptop pouches and tablet pouch

The final finished products are shown in Fig. 13. Laptop pouch A is developed by incorporating Warli art of Maharashtra with the traditional Kerala Mural painting. Laptop pouches B, C and tablet pouch E uses contemporary style of Kerala mural painting, ie, the mix of modern art with techniques of Kerala Mural painting. Laptop pouch D uses traditional Kerala Mural painting techniques with wordings to add a modern touch.

\section{Process Sequence for developing Shawl}

$2 \mathrm{~m}$ of Kasavu fabric was taken for shawl. The outline of the design was traced on the fabric using pencil. The yellow colour was applied first on the fabric as shown in Fig. 13. Second coating of yellow was given on the fabric. The colors were applied in the order of red, blue and green on the design as shown in Fig. 13. Final corrections were done for the painting. Painting was finished by using black outline (Fig. 14) and the raw edges of shawl were stitched.

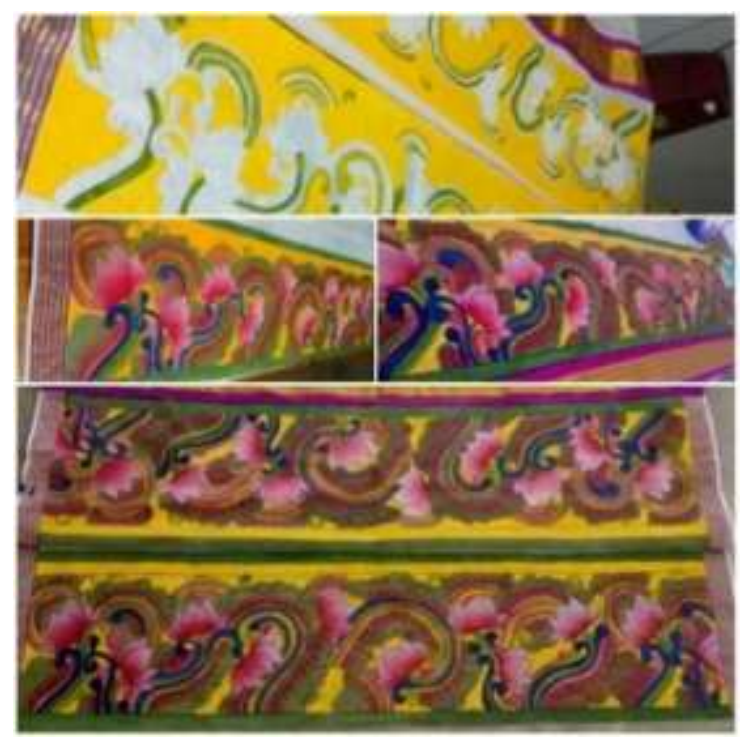

Fig.14: Process sequence of mural painting on shawl 
The final finished shawls shown in Fig 15 consisting of A, B, C, D and E were painting using traditional style of Kerala Mural painting.

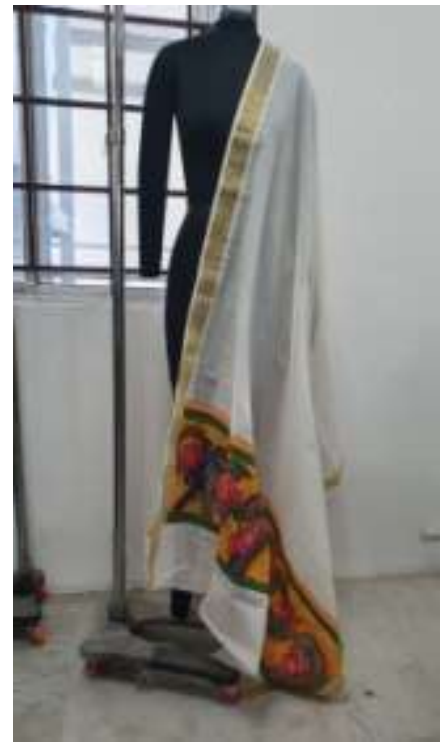

A

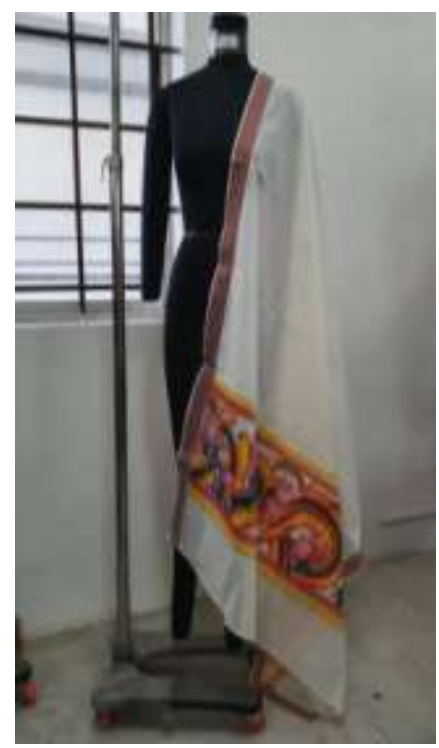

D

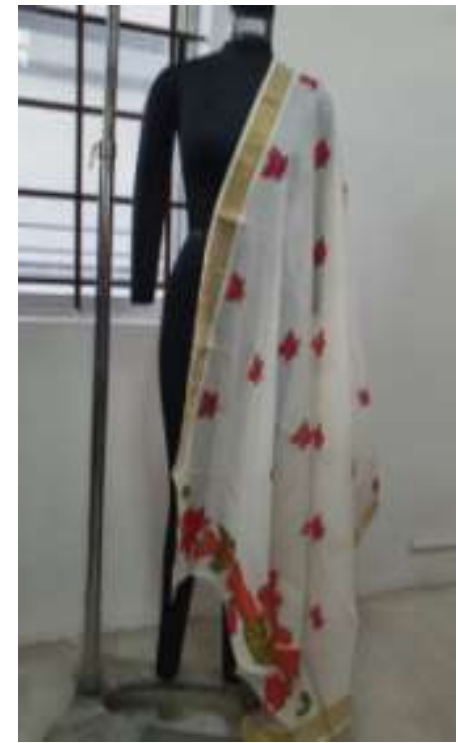

B

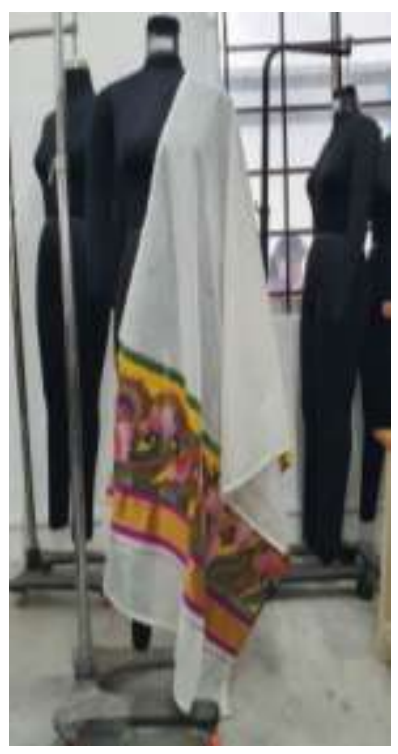

E

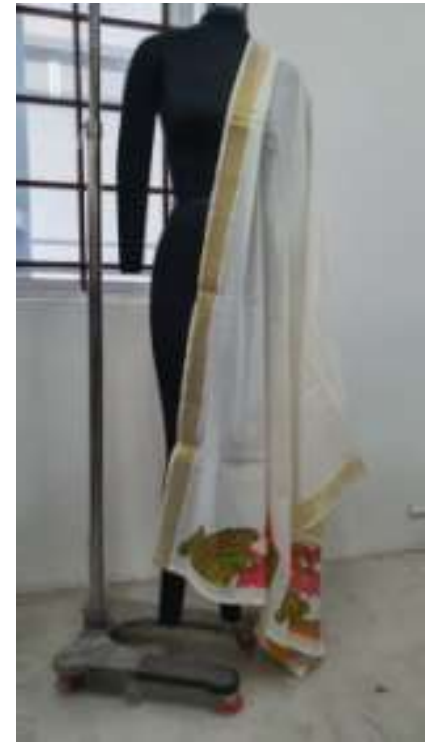

C

Fig 15: Developed shawls 


\section{Process Sequence for developing Wall hanging}

Canvas sheet was framed as shown in Fig 16.

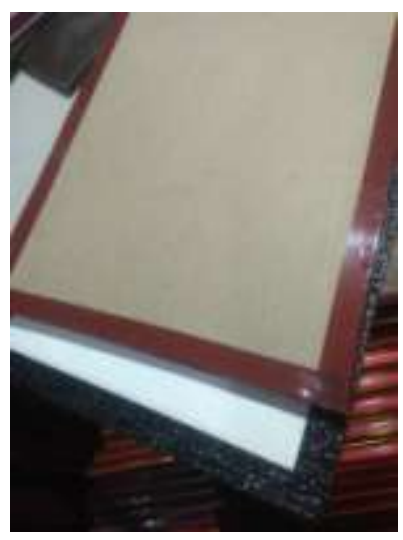

Fig.16: Framed canvas

Design was first drawn on a paper and then traced on the canvas using carbon paper. Yellow was applied first on the canvas (the order and choice of colours differs with the design). Final corrections were done for the painting. The painting was finished using black outline. Varnish was applied over the canvas for a longer life span. Fig. 17 shows the step by step process of developing the wall hangings.

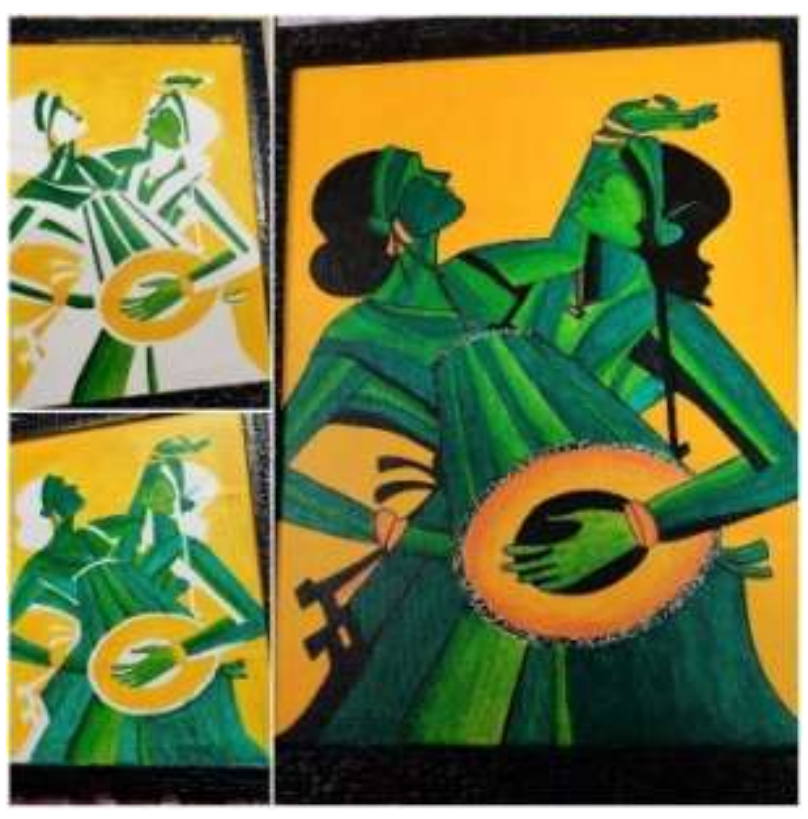

A

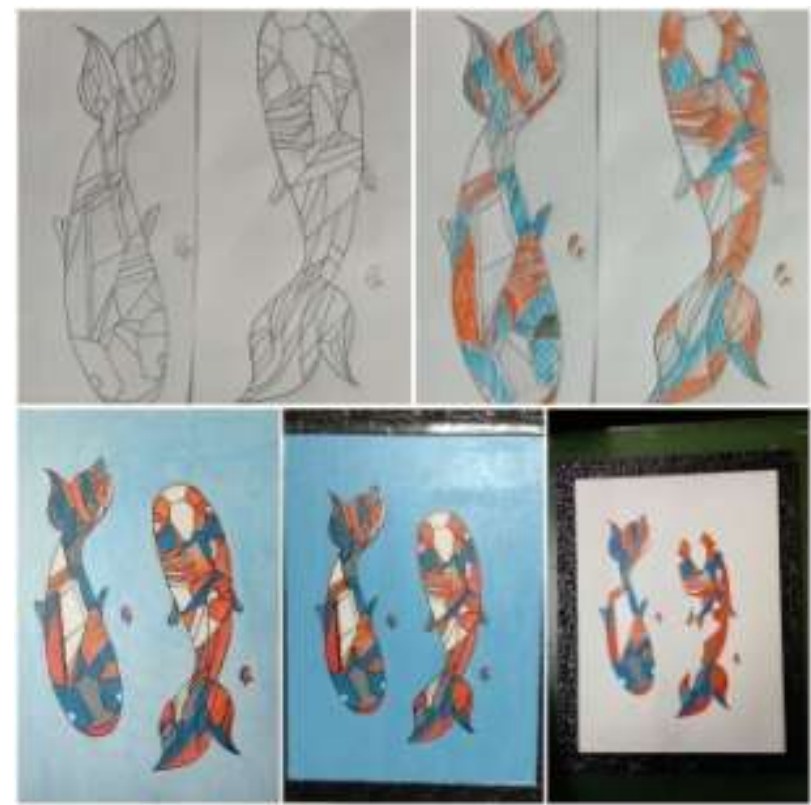

B 
13 | Kerala Mural Paintings: Diversification and Application in Home, Apparel and Accessories

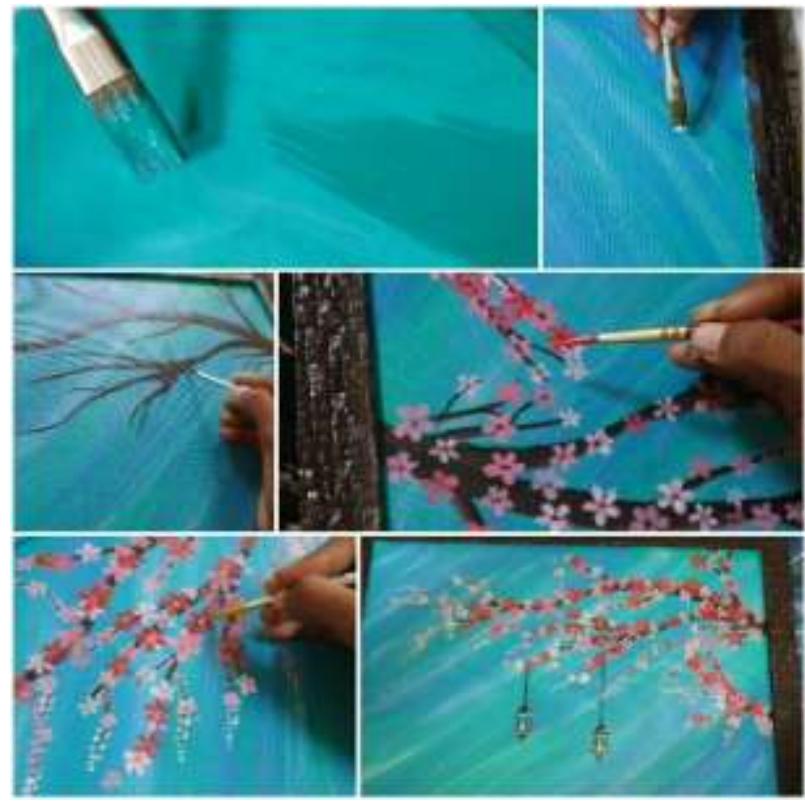

C

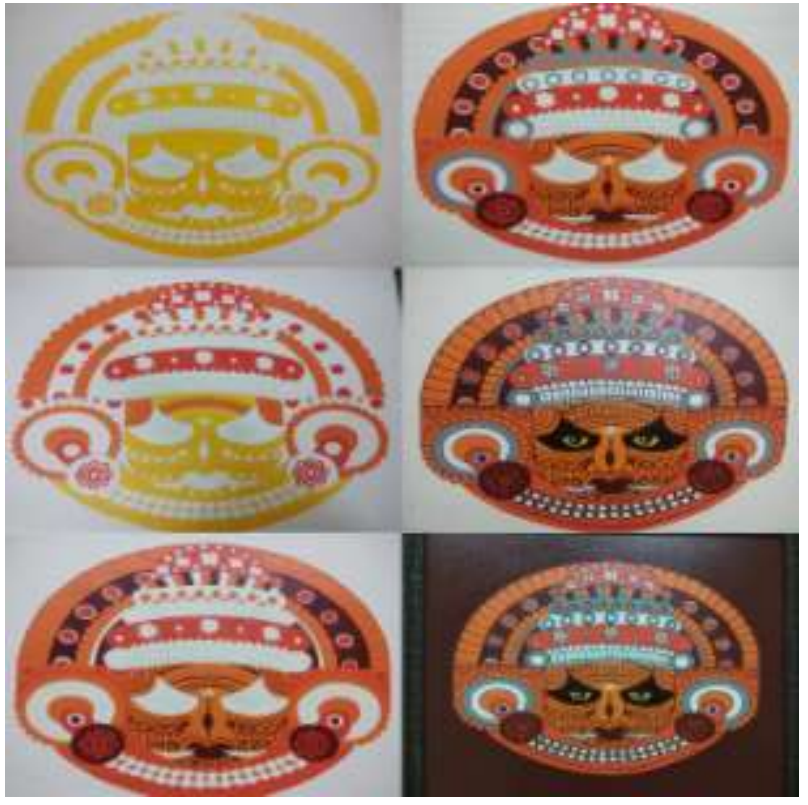

D

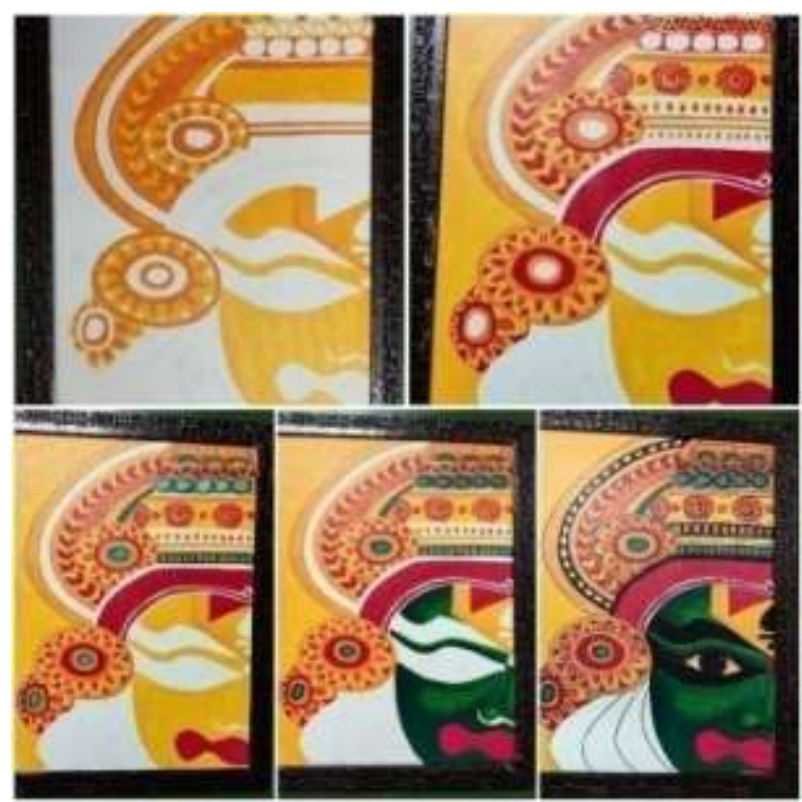

E

Fig 17: Process sequence of developing Wall Hangings 
The finished products can be seen in Fig 18.

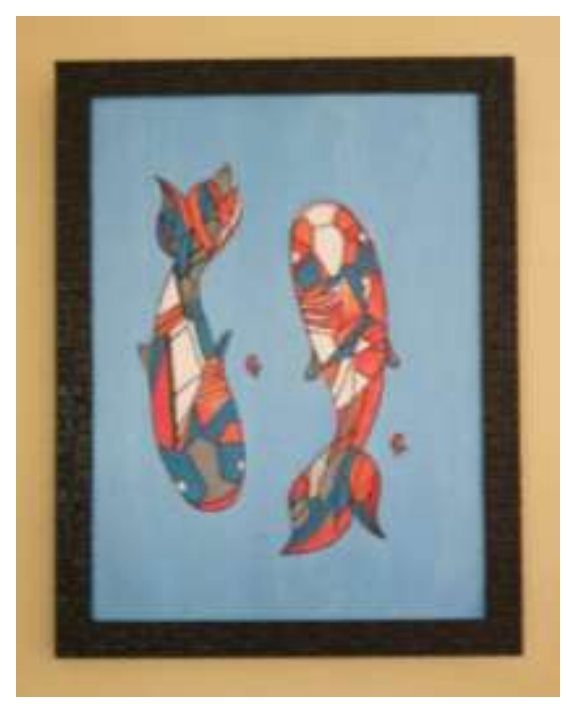

A

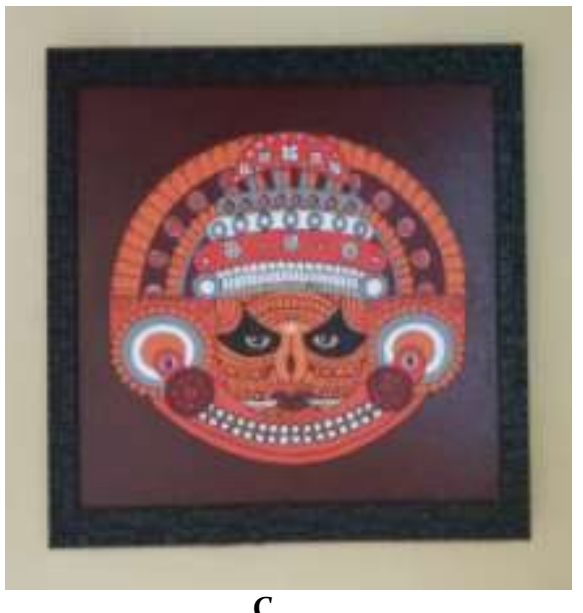

C

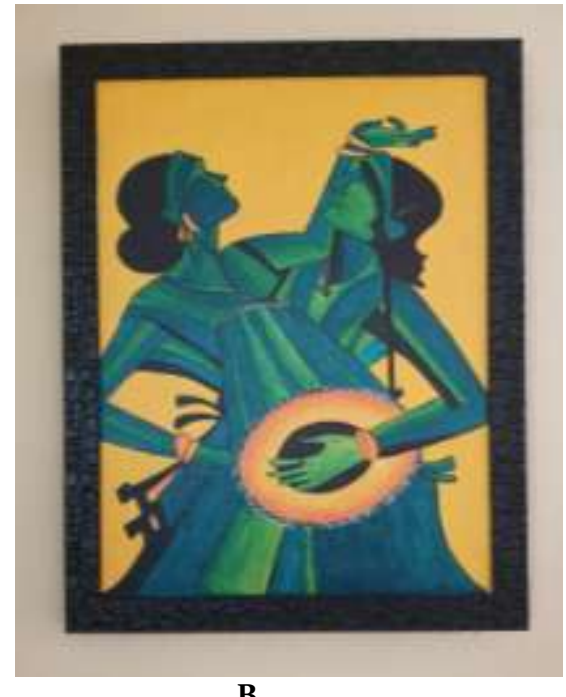

B

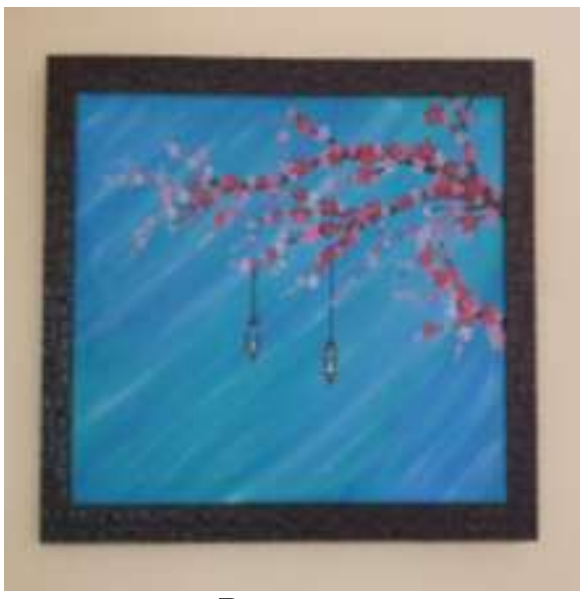

D

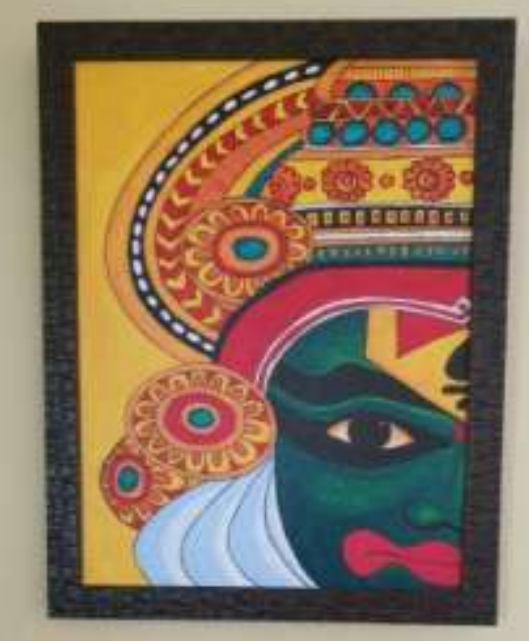


Fig 18: Developed Wall Hangings

As seen in the figure, wall hangings A, D and E uses techniques of traditional Kerala Mural painting, while $B$ and $C$ uses modern mural art technique.

\section{Evaluation on the developed mural products}

According to the survey, it was found that 50\%, 54\% and $76.6 \%$ of respondents rated excellent for the visual appearance of laptop pouch (A), shawl (B) and wall hanging (C) respectively as shown in Fig. 19.

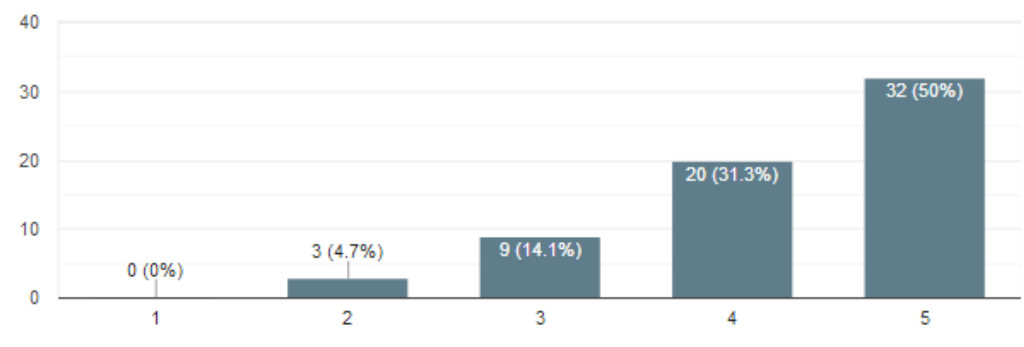

A
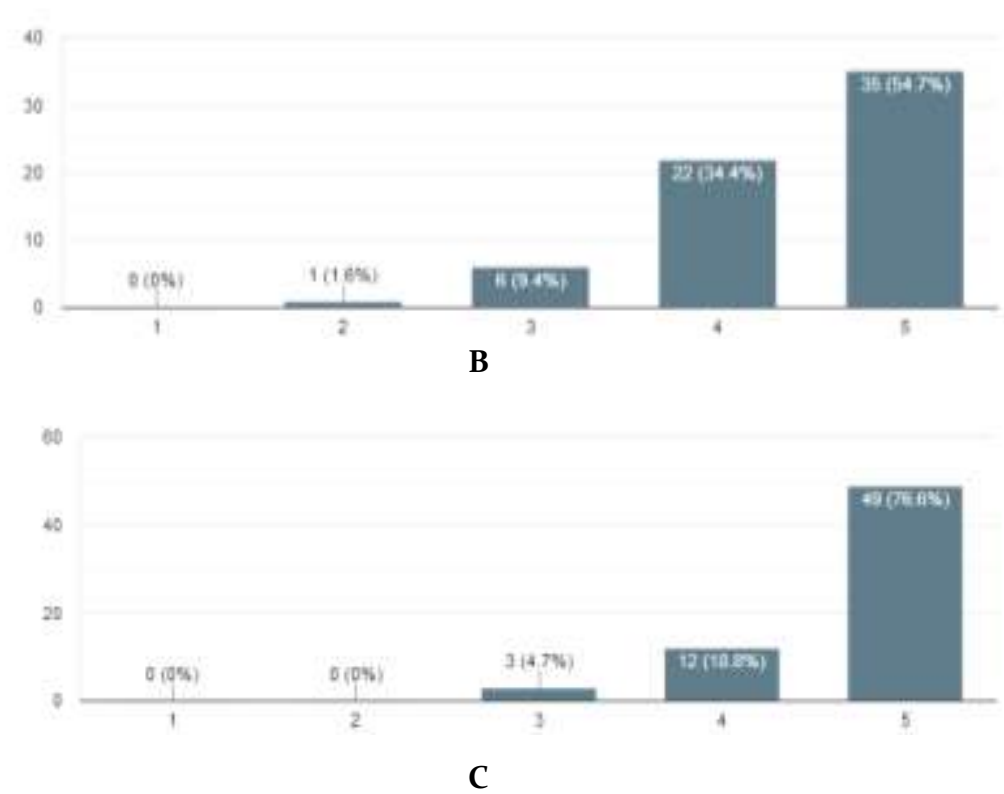

Fig. 19: Visual appearance of the developed products

With respect to design, about $82.8 \%$ of respondents liked the concept of fusion of Warli art of Maharashtra and Kerala mural painting done in laptop pouch.

According to the survey, $93.8 \%$ of respondents were willing to pay the price range of Rs.700 - 1000 for laptop pouch (A); $59.4 \%$ of respondents were willing to pay the price range of Rs.500 - 70o for shawl (B); $46.9 \%$ of respondents were willing to pay the price range of Rs.70o - 1000 for wall hanging $(C)$ as shown Fig. 20. 


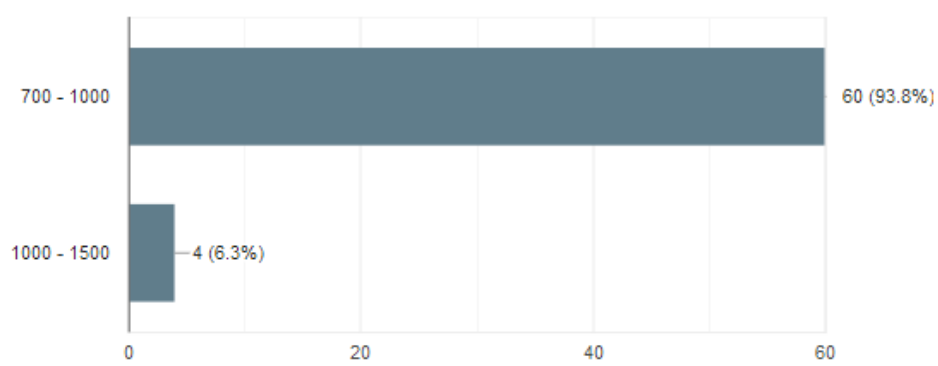

A
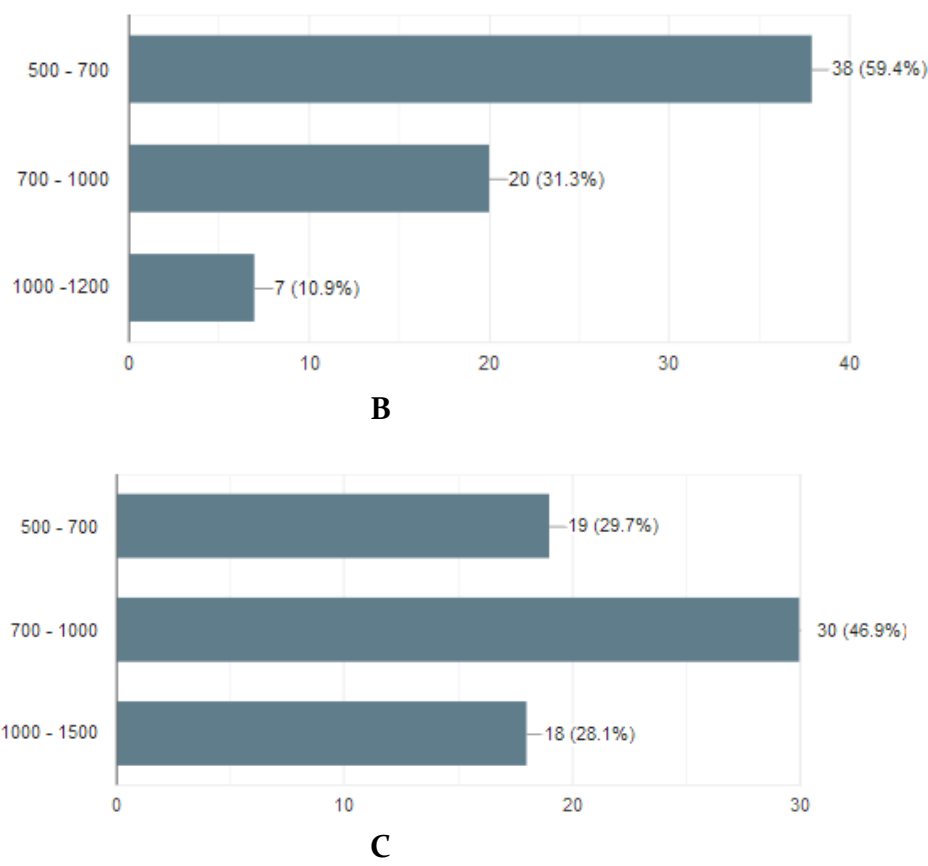

Fig. 20: Preferred price range of the developed product

\section{Pricing of the product}

The pricing of mural products such as laptop pouch, tablet pouch, shawl and wall hanging are mentioned below in table 3 .

Table 3: Pricing of the products

\begin{tabular}{|l|l|l|l|l|l|}
\hline \multirow{2}{*}{ S.no } & \multirow{2}{*}{ Particulars } & \multicolumn{4}{l}{ Price of the products (Rs.) } \\
\cline { 3 - 6 } & & Laptop pouch & Tablet pouch & Shawl & $\begin{array}{l}\text { Wall } \\
\text { Hanging }\end{array}$ \\
\hline 1. & & & & - \\
\hline 2. & Fabric & 50 & 50 & 244 & 74 \\
\hline 3. & Painvas and medium & 112 & - & - & 112 \\
\hline 4. & Sewing cost & 750 & 350 & 30 & - \\
\hline 5. & Framing cost & - & - & -112 & 350 \\
\hline 6. & Labour cost & 170 & 170 & 450 & 300 \\
\hline 7. & Profit 10\% & 108 & 68 & 84 & 84 \\
\hline
\end{tabular}




\begin{tabular}{|l|l|l|l|l|}
\hline Total price & 1190 & 750 & 920 & 920 \\
\hline
\end{tabular}

\section{Commercialization}

- Through exhibitions - Craft related exhibitions can be conducted like Craft Bazaars to showcase the mural products and its beauty to the public. More information about the mural paintings can be made known to the people who visit these exhibitions.

- Online websites -Having an online presence also helps to create awareness about the mural painting and also helps these mural paintings to have international exposure. Eg: Flipkart and Amazon.

- Retail shops - Collaboration can be made with retail stores in shopping malls to showcase, promote and sell these mural products.

Eg: Bhavm Murals.

\section{Suggestions for Craft Revival}

- Conducting Government funding workshops - Government funded workshops can be conducted for home maker and unemployed people to widen the opportunity of the craft.

- Street murals - Initiatives can be taken by college students and mural artisans to spread the awareness of the mural paintings through street art, wall art etc.,

- Online classes - Online classes can be conducted to spread the techniques of mural paintings known worldwide.

\section{Conclusion:}

Indian Mural paintings embarked its existence in Ajanta caves, Bagh caves and Sithanavasal caves and later evolved into various forms of murals across India. The reach of Mural paintings is wide and is found to be an irreplaceable art form in many states of India. Each state uses different techniques for Mural painting. Comparing all other states, Kerala Mural painting stands out for its vibrancy and elegance. The order of applying colour is one of the unique characteristics of Kerala Mural Painting (starting the painting with yellow colour followed by red, green, blue followed by black outline). We visited Bhavm Murals for studying the techniques that should be followed while developing a mural painting, the motifs of Kerala Murals, how these traditional paintings can be diversified and also developing various contemporary mural products. A survey was conducted to learn about the preferences of our potential customers and colleagues on Kerala Murals and products were developed based on this survey's results. The products included wall hangings, shawls and laptop pouches. These products were made using Kerala Mural painting techniques and also collaboration with other art forms. Later another survey was conducted to know the response of the same respondents about the developed products. The responses for the developed products were found positive. From this study, we have learnt that Kerala mural painting was known to all and were interested to buy not only traditional products but also diversified innovative products. 


\section{Acknowledgements}

We would like to thank Dr K. Prakasan, Principal, PSG College of Technology and Dr D. Vijayalakshmi, Professor and Head, Department of Apparel and Fashion Design, PSG College of Technology Coimbatore, for giving us the opportunity to carry out this work. We would also like to thank Mr Sujith, founder of Bhavm Murals, Wayanad for his vision and sincerity that helped us to learn the art and develop the diversified mural products.

\section{References}

Mini, P.V. (2010). Indian Journal of Traditional Knowledge, Preparation techniques of pigments for traditional mural paintings of Kerala, vol. 9(4)

Nimisha, P. (2017). Asian Journal of Multidisciplinary Studies, Mural paintings in Kerala - A Historical Overview, vol. 5

Sandhya Ravi (2015). International E-Journal of Advances in Social Sciences, Colour Culture and Identity: Influence of Colours on Kerala Mural Art, vol. 1 